\title{
COMMON FIXED POINTS FOR A CLASS OF COMMUTING MAPPINGS ON AN INTERVAL
}

\author{
J. CANO $^{1}$
}

\begin{abstract}
Let $C$ be a family of continuous commuting functions of an interval $I$ into itself. If each function, except for possibly one, has an interval $[a, b], a \leqslant b$, for its set of fixed points or does not have periodic points except fixed ones, then it is shown that $C$ has a common fixed point. This result generalizes a previous theorem of T. Mitchell.
\end{abstract}

1. Introduction. T. Mitchell [2] proved by means of topological semigroup methods that a family $F$ of commuting continuous self-maps of an interval such that for all $f \in F$, the iterates of $f$ form an equicontinuous family except for one possible exception, have a common fixed point. This was a generalization of $W$. Boyce's result [1], where only two functions were used. Both employed the techniques developed by A. Shields in [3].

In this note a larger class of functions is considered which has the common fixed point property and contains properly the class $F$ considered by Mitchell. This result is obtained by elementary means, and no use is made of topological semigroup methods.

2. Notation and terminology. All functions considered here are assumed to be continuous from the interval $I=[u, v]$ to itself.

Denote by $F_{f}$ and $P_{f}$ the set of fixed and periodic points of $f$ respectively, and by $L_{f}(x)$ the set of limit points of the sequence $\left\{f^{n}(x)\right\}_{n=0}^{\infty}$. Use is made of a result by Schwartz [4] to the effect that $L_{f}(x) \cap \bar{P}_{f} \neq \varnothing \forall x \in I$.

Define the classes of functions:

$$
\begin{aligned}
A & =\left\{f: I \rightarrow I \mid F_{f}=\left[a_{f}, b_{f}\right], a_{f} \leqslant b_{f}\right\}, \\
B & =\left\{f: I \rightarrow I \mid P_{f}=F_{f}\right\} .
\end{aligned}
$$

A class of functions $C$ is said to be an $H$-class if $C=C^{\prime} \cup\{h\}$ where $C^{\prime}$ is any subset of $A \cup B$ composed of commuting functions and $h$ is any function which commutes with the elements of $C^{\prime}$.

A class of functions $D$ is said to be an $F$-class if $D=D^{\prime} \cup\{h\}$ where $D^{\prime}$ is any family of functions such that the iterates of each element of $D^{\prime}$ form an equicontinuous family, and $h$ is any function that commutes with the elements of $D^{\prime}$.

Received by the editors October 8, 1981 and, in revised form, March 24, 1982.

1980 Mathematics Subject Classification. Primary 54H20.

' The author thanks the referee for improving this article. 


\section{Results.}

THEOREM 1. There is a common fixed point for every $H$-class in $I$.

Proof. Let. $C$ be an $H$-class, and $C_{1}$ a finite subset of $C . C_{1}$ can be written as

$$
C_{1}=\left\{a_{1}, \ldots, a_{n}\right\} \cup\{h\} \cup\left\{b_{1}, b_{2}, \ldots, b_{m}\right\}
$$

where $a_{i} \in A, i=1, \ldots, n, h$ is a possible arbitrary function that commutes with the elements of $C^{\prime}, b_{j} \in B, j=1, \ldots, m$. Since $F_{a_{i}}$ is an interval and the $a_{i}$ 's commute, $\bigcap_{i=1}^{n} F_{a_{i}}$ is an interval $[a, b]$. Also by the commutativity of $h$ with the $a_{i}$ 's, $h$ takes $[a, b]$ into $[a, b]$, and so it must have a fixed point $z \in[a, b]$. Now $\left\{b_{1}^{n}(z)\right\}_{n=0}^{\infty}$ has a limit point $z_{1} \in F_{b_{1}}$ since $P_{b_{1}}=F_{b_{1}}$ and $F_{b_{1}}$ is a closed set. But $z_{1} \in \bigcap_{i=1}^{n} F_{a_{1}} \cap F_{h}$ because $b_{1}$ takes $\cap_{i=1}^{n} F_{a_{1}} \cap F_{h}$ into itself. Similarly $\left\{b_{j}^{n}\left(z_{j-1}\right)\right\}_{n=0}^{\infty}, j=2, \ldots, m$, has a limit point $z_{j}$ which is fixed for $b_{j}, \ldots, b_{1}, h, a, \ldots, a_{n}$; thus $\cap F_{f} \neq \varnothing \forall f \in C_{1}$. Now since $I$ is compact, $\cap F_{f} \neq \varnothing \forall f \in C$. The cases where $C$ contains no such $h$, $C \cap A=\varnothing$, or $C \cap B=\varnothing$ are clear from the above proof.

THEOREM 2. Let $f$ be a function such that its iterates form an equicontinuous family. Then

(1) $f \in A$,

(2) $f \in B$ if $F_{f}$ is nondegenerate.

Proof. If $F_{f}$ is a singleton, we are done. So suppose there are $a, b \in F_{f}, a<b$ and $\forall x \in(a, b), x \notin F_{f}$. Then $f(x)>x$ or $f(x)<x$. Assume $f(x)>x \forall x \in(a, b)$ (the case $f(x)<x$ is done similarly).

We consider two cases.

(i) $f(x)<b \forall x \in(a, b)$,

(ii) $\exists x \in(a, b) \exists f(x) \geqslant b$.

Case (i). $\forall x \in(a, b),\left\{f^{n}(x)\right\}_{n=0}^{\infty} \rightarrow b$ and so the family of iterates of $f$ cannot be equicontinuous at $a$.

Case (ii). Let $z$ be the smallest point in $(a, b)$ with $f(z)=b$. There is a sequence $\left\{x_{n}\right\}$ in $(a, z]$ such that $\left\{x_{n}\right\} \rightarrow a, x_{1}=z$ and $f\left(x_{n}\right)=x_{n-1}$. Thus $f^{n}\left(x_{n}\right)=b$ for $n=1,2, \ldots$ and the family of iterates of $f$ cannot be equicontinuous at $a$ also. This contradiction establishes part (1).

For part (2), let $F_{f}=[a, b]$ with $a<b$. Suppose $f^{n}(x)=x$ for some $n$ and some $x \in[u, a)$ (the case $x \in(b, v]$ is similar). Applying part (1) to the function $f^{n}$, we obtain $f^{n}(y)=y \forall y \in[x, a]$. But since $f(y)>y \forall y \in[u, a)$, and $f(a)=a$, we may choose $y \in(x, a)$ close enough to $a$ so that either $y<f^{n}(y)<a$ or $y<f^{m}(y)$ $\in[a, b]$ for some $m \leqslant n$. Then $f^{n}(y)>y$, a contradiction.

For $I=[0,1]$ the function $f(x)=-x+1$ shows that the condition that $F_{f}$ be a nondegenerate interval is necessary. The class of functions $\left\{x, x^{2}, x^{3}, \ldots\right\}$ is an $H$-class that is not an $F$-class on $[0,1]$. 


\section{REFERENCES}

1. W. Boyce, On Г-compact maps on an interval and commutativity, Trans. Amer. Math. Soc. 160 (1971), 87- 102 .

2. T. Mitchell, Common fixed points for equicontinuous families of mappings, Proc. Amer. Math. Soc. 33 (1972), 146-150.

3. A. Shields, On fixed points of commuting analytic functions, Proc. Amer. Math. Soc. 15 (1964), 703-706.

4. A. Schwartz, Common periodic points of commuting functions, Michigan Math. J. 12 (1965), 353-355.

Departamento de Matematicas, Universidad Simon Bolivar, Caracas, Venezuela 